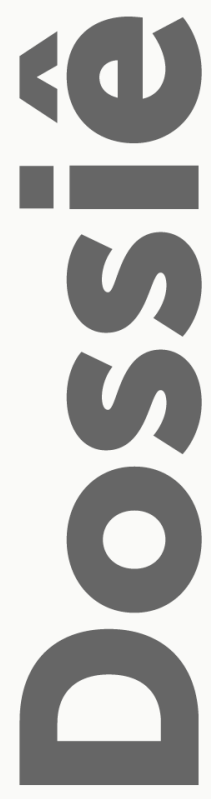

\title{
La muerte en la Institución hospitalaria
}

\author{
Death in the hospital Institution
}

\section{RESUMEN}

El presente texto tiene como objetivo problematizar la muerte y la enfermedad en el contexto hospitalario del estado de Puebla, México, en las dos primeras décadas del siglo XXI. Para cumplir esta tarea, se han sistematizado las experiencias de tres intervencionesinvestigaciones diferentes. No obstante, el material recuperado de los diarios de campo, de las observaciones participantes y de las entrevistas de estas tres investigaciones se ha organizado en torno a las categorías siguientes: muerte en el contexto hospitalario, procesos agónicos y enfermedades terminales. Por lo que será a partir de esta sistematización de experiencias que se propone un recorrido que va del pasado religioso del hospital hasta su consolidación como institución clínica, tomando como supuesto que, en dicho proceso de institucionalización, se ha tendido a negar y a ocultar fenómenos claves de la vida social, como la muerte y la enfermedad. Para lograr estos objetivos recuperamos los trabajos arqueológicos y genealógicos de Michel Foucault, en particular aquellos sobre los procesos de construcción de dispositivos sociales clínicos.

Palabras clave: Muerte - Hospital - Institución - Psicología Hospitalaria - Duelo

\begin{abstract}
The objective of this text is to problematize death and disease in the hospital context of the state of Puebla, Mexico, in the first two decades of the 20th century. To accomplish this task, we systematized the experiences of three different research projects. The material recovered from field diaries, participant observations and interviews from the research projects has been organized around the following categories: death in the hospital context, dying processes, terminal illnesses. The systematization of experiences results in an analysis that goes from the religious past of the hospital to its consolidation as a clinical institution, assuming that, in the processes of institutionalization, key phenomena of social life, such as death and disease, are denied and hidden. In order to achieve the research objectives, we recover Michel Foucault's archaeological and genealogical works, particularly those focused on the processes of construction of clinical social devices.
\end{abstract}

Keywords: Death - Hospital - Institution - Hospital Psychology Grief

* Doctorado en Sociología en el Instituto de Ciencias Sociales y Humanidades "Alfonso Vélez Pliego" de la Benemérita Universidad Autónoma de Puebla. Docente en la Licenciatura en Psicología del Instituto de Estudios Avanzados Universitarios (IDEAUNI), con incorporación (8955-25) a la Universidad Nacional Autónoma de México. Puebla, México. CV: https://buap-mx.academia.edu/MayZam

** Profesor-investigador del Departamento de Educación y Comunicación de la Universidad Autónoma Metropolitana-Xochimilco. CV: https://sia.xoc.uam.mx/sia/profesor_investigador/resp.php?index=7402 
a muerte en el contexto hospitalario es un tema cotidiano pero invisibilizado en la sociedad actual. No obstante, a lo largo de diversas intervenciones desde la psicología social de la salud - comunitaria e institucional -, se ha recuperado una serie de observaciones, entrevistas e información proveniente del personal de salud, familiares y pacientes que han mirado a la muerte y sus procesos agónicos directamente a los ojos. Bajo las inquietudes sobre ¿cómo convivimos con la muerte en contexto hospitalario actual? El presente texto tiene como objetivo problematizar la muerte y la enfermedad en el contexto hospitalario del estado de Puebla, México.

Se debe aclararque toda la información presentada, pertenece a diferentes intervenciones y no a una única investigación; a partir de las cuales, se sistematizó la información referente a cómo se experimenta la muerte, la enfermedad y los procesos agónicos en la institución hospitalaria. Se debe destacar que, en todas las intervenciones se contó con autorizaciones verbales -y de algunos casos por escrito- de parte del personal de salud, familiares y pacientes, para hacer uso de sus datos con fines investigativos. No obstante, se conserva el anonimato de todos los participantes.

Los primeros acercamientos con el campo hospitalario fueron en el mes de febrero del año 2009, en el área de urgencias en el Instituto de Seguridad Social y Servicios Sociales de los Trabajadores al Servicio del Estado de Puebla - ISSSTEP -, ubicado en la Ciudad de Puebla, México. Lugar donde una de nosotros, Mayleth A. Zamora, realizaba prácticas de psicología hospitalaria y de enlace para una certificación en "Intervención en Crisis" de parte de la Cruz Roja Mexicana. En esta primera intervención, se realizó una observación participante, ${ }^{1}$ que es una técnica de corte cualitativo que tiene la virtud de permitirle al investigador conocer directamente el contexto sociohistórico, en el cual tienen lugar las articulaciones de los sujetos, facilitando el acceso al conocimiento, marco simbólico y cultural de las personas en su contexto cotidiano (Guber, 2005, p. 170-182).

Esta técnica consiste en dos actividades, la primera se trata de observar sistemática y controladamente el universo y la población de la investigación. En ese sentido, se supone una construcción del "campo" de parte del investigador. En otras palabras, una delimitación clara del tiempo y lugar de dónde se realizará el registro de investigación. La segunda actividad, consiste en interactuar directamente con la población que se investiga. Por lo que no se reduce a una observación directa, donde el investigador no puede dialogar con los sujetos. Por el contrario, la observación participante le exige al investigador sumergirse subjetivamente en el contexto que está investigando (Guber, 2005, p. 109). Todas las observaciones de esta intervención fueron registradas en un diario de campo. Dicha herramienta implica un registro sistemático de información, delimitada por los objetivos de la investigación.

El segundo encuentro con la muerte y la enfermedad en el contexto hospitalario fue durante el trabajo de campo de la investigación de maestría en Psicología Social de Grupos e Instituciones. ${ }^{2}$ La intervención tuvo lugar en el Hospital Universitario de Puebla. A través de observación participante, entrevistas libres y registros en diarios de campo, se recuperó una serie de experiencias de la vida, quehacer y praxis en el hospital en el periodo de septiembre del 2015 a febrero del 2016. 
Las áreas hospitalarias involucradas en este trabajo fueron: urgencias, medicina interna, unidad de cuidados intensivos y quirófano. Durante esta intervención se recuperaron experiencias y anécdotas de un total de 47 médicos de diferentes grados y áreas: 7 adscritos (hombres y mujeres) a las áreas de urgencias, medicina interna y cirugía; 20 residentes (hombres y mujeres), 3 de ortopedia, 3 de cirugía, 2 de gineco-obstetricia y 12 de medicina interna, todos de diferentes años de residencia (del primer año al cuarto). ${ }^{3}$ Cabe señalar que las entrevistas y observación participante que se realizaron durante esta investigación estaban dirigidas a problematizar y reflexionar sobre las experiencias del personal de salud que trabajaba en este hospital.

La tercera intervención, se llevó a cabo gracias a un proyecto de prácticas académicas y de servicio social de la licenciatura en Psicología, en el periodo de marzo a junio del 2017.4 Durante esta se recuperaron las experiencias del personal de salud, de pacientes hospitalizados y sus cuidadores del Hospital General de Tepeaca, Puebla. Mayleth A. Zamora participó como coordinadora de la licenciatura y parte del equipo de investigación con otros dos psicólogos.

Los objetivos de esta intervención estaban orientados a la identificación del papel de la psicología hospitalaria en la adherencia al tratamiento y en la disminución del uso de analgésicos de los pacientes hospitalizados. Los instrumentos utilizados para la recolección de datos consistieron en: observación participante; diarios de campo de alumnos, profesores supervisores y de la coordinadora del proyecto; entrevistas estructuradas y semiestructuradas con pacientes, familiares y personal de salud; y pruebas psicométricas y técnicas proyectivas encaminadas a la detección de malestar y sufrimiento psíquico de los pacientes, en relación a la hospitalización e intervención médica.

Para este artículo, se apeló como propuesta metodológica, a la sistematización de experiencias. Dicho instrumento permite agrupar una serie de ideas, acontecimientos y situaciones de prácticas sociales concretas, desde la premisa según la cual las experiencias son procesos sociales dinámicos que se encuentran en permanente cambio y movimiento (Curso de Sistematización de Experiencias, 2015).

La sistematización de experiencia, en tanto herramienta de corte cualitativo, consiste en organizar los contenidos, actividades e información de los sujetos, con el fin de analizarlas bajo categorías y conceptos en común (Curso de Sistematización de Experiencias, 2015). Además, implica:

La interpretación crítica de una o varias experiencias, que, a partir de su ordenamiento y reconstrucción, descubre o explica la lógica del proceso vivido, los factores que han intervenido en dicho proceso, cómo se han relacionado entre sí y por qué lo han hecho de ese modo (Jara cit. en Curso de Sistematización de Experiencias, 2015, p. 15).

De tal manera que todo el material recuperado de los diarios de campo, de las

3 La residencia es el nombre coloquial para los alumnos de posgrados y especialidades médicas.

4 Instituto de Estudios Avanzados Universitarios (IDEAUNI) con incorporación a la Universidad Nacional Autónoma de México. Clave de incorporación 8955-25. 
observaciones participantes y de las entrevistas, de las tres investigaciones mencionadas, se ha organizado en torno a las categorías siguientes: muerte en el contexto hospitalario, procesos agónicos y enfermedades terminales.

A partir de la sistematización de experiencias surge la inquietud de problematizar y reflexionar sobre la muerte en el contexto hospitalario. Por ello nos preguntamos: ¿realmente la muerte es tan poco ruidosa e higiénica como declara Norbert Elías (2015) en la Soledad de los Moribundos?; o, como afirma Iván Illich (1975) en la Némesis médica, en la sociedad actual ¿las tecnologías médicas están dirigidas a desaparecer el dolor, ocultar la enfermedad y abolir la agonía y la muerte?

Para ello proponemos dos aproximaciones complementarias: por una parte, un recorrido que va del pasado religioso del hospital hasta su consolidación como institución clínica, tomando como supuesto que, en dicho proceso de institucionalización, se ha tendido a negar y a ocultar fenómenos claves de la vida social, como la muerte y la enfermedad; por otra parte, reconocemos que es posible visibilizar los fenómenos señalados a través de experiencias y elaboraciones del personal de salud, familiares y pacientes, que han convivido con la muerte y la enfermedad en el contexto hospitalario.

Para lograr estos objetivos recuperamos los trabajos arqueológicos y genealógicos de Michel Foucault, en particular aquellos sobre los procesos de construcción de dispositivos sociales, los cuales nos permiten preguntarnos: ¿cómo se ha configurado históricamente una serie de tecnologías diseñadas para el control, direccionamiento y ejercicio de ciertos discursos y prácticas sociales? En específico, las tecnologías que han estado orientadas a la construcción de la institución clínica-hospitalaria.

\section{Del hospicio al hospital}

John Berger (2011), señala que antes del arribo de la modernidad, los vivos, esperaban alcanzar la experiencia de los muertos. La muerte se miraba como un futuro dado, irremediable. Los vivos se miraban a sí mismos como incompletos hasta alcanzar su destino irremisible. En ese sentido, existía una interdependencia entre vivos y muertos.

No nos detendremos demasiado en este punto, lo que nos interesa destacar es la mediación simbólica que produce la pregunta e inquietud sobre la muerte, y lo que suponemos, articula el tejido social (Girard, 2005; Le Bouhellec, 2017). En ese sentido, lo religioso, en su connotación más esencial del religare, se convierte en una mediación de significaciones y significados que, a través de las tradiciones, usos, costumbres, lógicas y dinámicas, religa a los sujetos unos con otros bajo las interrogantes sobre la vida y la muerte (Matamoros Ponce, 2004).

La Iglesia, como institución, es decir como "la red simbólica, socialmente sancionada en la que se combinan en proporción y en relación variables un componente funcional y un componente imaginario" (Castoriadis, 1983, p. 227-228), se convierte en la mediadora por excelencia de las relaciones entre los sujetos con la vida, la finitud y el más allá. Bajo esa 
lógica, el hospicio, -como parte del engranaje religioso-, será el espacio simbólico e imaginario destinado para la asistencia a los pobres en espera de la muerte (Foucault, 1996, p. 73; Donzelot, 2008, p. 34).

Hasta antes del siglo XVIII, la atención médica era reservada a una práctica individual y accesible sólo para la clase dominante. El resto de la población -lumpen-, estaba excluida de la intervención médica. Para ellos, la terapéutica estaba a cargo del personal eclesiástico, implicando que más allá de tratarse de una intervención sobre el cuerpo, se trataba de una sobre la salvación del alma (Foucault, 1996, p. 73; Donzelot, 2008, 34). Esta práctica se profesaba en el hospicio, conviertiéndose en un lugar para la confesión del pecado y de la extremaunción, que lejos de buscar la curación, el personal eclesiástico brindaba escucha y acompañamiento en la recta final de la vida de los pobres.

Hasta la mitad del siglo XVIII nadie salía del hospital. Se ingresaba en estas instituciones para morir. La técnica médica del siglo XVIII no permitía al individuo hospitalizado abandonar la institución en vida. El hospital representaba un claustro para morir, era un verdadero mortuorio (Foucault, 1996, p. 73).

Sin embargo, con el despliegue industrial capitalista ubicado tradicionalmente con la Revolución industrial inglesa, y con la configuración de los Estados-Nación modernos, - el cual es referido a partir de la Revolución Francesa (Rifkin, 2004) -, se configurarán procesos de transformación social, que en sí mismos se traducirán en otras formas de relación social. En este caso, el papel de la institución hospitalaria.

Con la Revolución Francesa, se cuestionan todos los "fundamentos" sostenidos hasta el momento: de los ciudadanos, de los derechos, la religión, la libertad de los individuos, y con ello, de la salud de los mismos. Esta ruptura devino en una sensación de que todo "mal" ha sido eliminado, como si se volviera a un estado de salud originaria, la cual, puso sobre la mesa a la medicina como una entidad pasajera (Donzelot, 2008, p. 19-52; Foucault, 2014). ${ }^{5}$ Por medio de los ideales revolucionarios, el individuo tendría que hacerse cargo de su salud. Ahora la tarea del médico era encaminar este propósito.

Así, la autoridad médica, ya no solamente se tratará de una autoridad de saber, como aquella persona erudita sobre las enfermedades (Foucault, 1998), sino se convierte en una autoridad social que deberá tomar decisiones sobre la ciudad, el barrio, la institución y los reglamentos. Aparece un campo de intervención de la medicina diferente al de las enfermedades: "el aire, el agua, las construcciones, los terrenos, los desagües" (Foucault, 1996, p. 77). Y con ello, se introduce un aparato de medicalización colectiva: el hospital. Pero ahora, ya no como mortorio, sino como una entidad encaminada a lograr que ciertos enfermos salieran de éste.

Es así, que el hospital se aleja de su pasado religioso y comienza a ingresar a los albores de la modernidad. En la medida que éste se configura con el nuevo papel de la medicina como autoridad de regulación y control social; el hospicio-hospital pasa de ser un lugar para

5 Con respecto esta discusión, ya se ha trabajado más a fondo sobre el papel de la familia como agente-puente entre la autoridad médica y el Estado. 
el recibimiento del enfermo, el pobre y de su muerte, a uno de ortopedia, curación y reclusión (Foucault, 1996). ${ }^{6}$

Con la modernidad, pareciera que se desvaneciera el universo de lo simbólico-religioso para darle paso a la razón; ${ }^{7}$ las utopías religiosas se secularizan. Se trata del paraíso en la tierra, el hombre se convierte en el nuevo dios, y que mejor ejemplo materializado que el médico, quien comienza a trascender la muerte (Foucault, 1996, 2014). Bajo este marco utópico, el cuerpo se convierte en parte de esta maquinaria de producción, bajo la ilusión de que "ya no hay límites". Este no-límite se encarnará bajo las prácticas del médico francés Bichat, quién a través de la necropsia condensará toda lógica moderna y la ruptura con el marco simbólicoreligioso previo (Foucault, 2014, p. 198).

En opinión de Foucault (2014, p. 198), este momento representará un parteaguas, ya que él "liberará" a la medicina del miedo a la muerte integrándolo a un conjunto técnico y conceptual. En palabras de Bichat: "abrid algunos cadáveres: veréis desaparecer enseguida la obscuridad que la observación sola no ha podido disipar". No obstante, este no fue el único paso o movimiento que produce "la ruptura" por sí mismo, sino que estuvo articulado con la introducción de los mecanismos de la administración médica, -en su cara de "medicina social"-, abrieron las posibilidades sobre la intervención patológica a través del hospital y del cuerpo (Foucault, 1996). Es decir, en la medida en que la medicina se convirtió en una práctica social, como dispositivo organizador de la vida social, y dejó de ser exclusivamente una práctica individual.

De este modo, el trabajo de médicos como Bichat, Laennec y Bayle, encarnarán la máxima ruptura con el modelo simbólico-religioso pre-moderno, que a través de la práctica de la transgresión e intervención sobre el cuerpo humano muerto, se configurará a la muerte como un espejismo, un semblante que deja de ser un espacio inaccesbile para el ser humano. Ahora, al menos en apariencia, es asible. El cadaver se articula con el propósito científico, en favor de la ciencia y el progeso, del "bien común" (Foucault, 1996).

En ese sentido, el discurso de la clínica se materializará no solamente con la urbanística, sino a tarvés del cuerpo físico. ${ }^{8}$ Las técnicas de la medicina consituirán al sujeto moderno. Por un lado, con la mirada, y por el otro, con el lenguaje. La clínica se corporizará por medio de la tecnología hospitalaria, la cual, funcionará como un espacio pedagógico, de conocimiento y de comunicación, un lugar de observación constante, donde se codifica y sistematiza al individuo. Éste operará como un instrumento de verificación del saber a través de la mirada. (Foucault, 1996, 2014).

"La clínica hospitalaria permite [...] poner aparte lo extrínseco" (Foucault, 2014, p. 153). Esto es, da la opción por medio de la observación clínica de no escuchar al paciente, sino que

6 Recordemos que los espacios hospitalarios se configurarán bajo las lógicas de las prácticas divisorias, en la producción de otredad y del encierro (Foucault, 1996, 1998, 2007). Esto será vital para la conformación de la tecnología hospitalaria como parte del control social.

7 Aunque, al mirar con atención, se trasluce un paso a la religión científica, a una Fe en la objetividad y a la razón pura (Rifkin, 2004).

8 Las prácticas del control del cuerpo social en su carácter del demos y del cuerpo individual del zoé, se entretejerán perfectamente con el discurso de las ciencias. Consecuencia lógica y directa del proyecto de la modernidad y que simultáneamente lo legitima, respalda y reivindica. 
por medio del saber y de la técnica, el paciente se convierte en un receptáculo de la patología que sólo confirma lo ya observado. El paciente es sólo un objeto que hay que conocer. Así, la clínica, que cobrará forma a través de la institución-hospital, se apropiará del derecho de hacer uso de un discurso de poder. Asume una posición, el estatuto del médico, comportándose bajo criterios de competencia y de saber; instituciones sistemas, normas pedagógicas, condiciones legales que dan derecho, que fija límites, tanto a la práctica como a la experimentación del saber sobre el cuerpo poblacional y el individual, esto es un dispositivo (Foucault, 2013, 2014).

\section{El hospital como institución}

La historia del paso del hospicio como entidad religiosa, como espacio social de muerte y agonia, hacia un espacio de producción de saber y de regulación social, nos habla de reconfiguración de las relaciones sociales.

En este proceso, el hospital se torna forma institucional de equivalencia recíproca entre la impiedad, la locura y la pobreza (Focault, 1996). Es decir, el hospital, en un carácter de institución-encierro y de tecnología de producción de saber-poder, se configura como un dispositivo regulador de la sociedad, estableciendo otras formas de relacionarse con la enfermedad y la muerte. Por ejemplo, el considerable progreso médico y su intervención sobre el cuerpo para sostener la vida, se consolidó en los años 1844-1847 con el descubrimiento de los analgésicos y anestésicos. Con ellos, ahora se podía adormecer no sólo el dolor, sino a la persona, al cuerpo, con el fin de practicar una intervención quirúrgica (Foucault, 1996).

Al desaparecer la barrera del dolor y el sufrimiento, ahora se puede proceder a cualquier operación. En la misma línea se encuentra la introducción de la asepsia en 1879, la cual implicó el mejoramiento de las condiciones higiénicas disminuyendo así la morbilidad en los quirófanos (Foucault, 1996). De esta forma, la institución hospitalaria comenzará a cobrar sus componentes imaginarios contemporáneos, el hospital se convierte en un espacio de curación. Este espacio de entrecruzamiento institucional -pedagógico, de encierro, clínica-, se convierte en un lugar de confrontación del saber. Por un lado, al estar ingresado en el establecimiento hospitalario, se retira al enfermo de su medio, con el fin de aislarlo de aquello que pueda engañar o confundir a la mente; por el otro, se le confina bajo ciertos estatutos y límites para producir verdad a través del dispositivo clínico (Foucault, 1996, 2014).

Bajo este marco, se constituirán los cimientos de lo que se entenderá por salud, la enfermedad y la muerte. Se trata de nuevas dimensiones de las tecnologías médicas, - las que Foucault denomina la "cuestión de la biohistoria". El biólogo y el médico se articulan para trabajar en torno a la vida de los sujetos, y ya no de la muerte (Foucault, 1996, p. 74). En otras palabras, hay un paso del arte del acompañamiento en la agonía y la enfermedad, a la salvación de la vida. ${ }^{9}$ En ese sentido, en las sociedades modernas, la institución hospitalaria surge en el contexto de un dispositivo social destinado a hacerce cargo de la salud de una población.

9 Todavía en la segunda mitad del s. XIX. Gubler, siguiendo a Bérard, planteaba que el papel de la medicina era "curar pocas veces, aliviar a menudo, consolar siempre" (Fresquet Febrer, 1993). La intervención terapéutica del médico tuvo una evolución muy veloz a partir de entonces. 
Empero, como efecto paradójico, se ocultan la enfermedad y la muerte detrás de los muros hospitalarios, alejándolas de la vida cotidiana y del ámbito social (Emmer y Schejter, 2014, s. p.).

Este efecto paradójico trae consigo una serie de problemáticas que no siempre están visibilizadas. Al abordarlo desde la sistematización de experiencias del lado de los familiares y de los pacientes, escuchamos claramente una demanda de "curación", es decir, acuden al hospital para ser atendidos desde una terapéutica que sane y resuelva su malestar. Por otro lado, se espera una respuesta desde el lugar de saber, el médico debe indicar qué es lo que tiene, cómo se trata, qué esperar en el desarrollo de la enfermedad, etcétera.

Desde el lugar del médico, en muchas ocasiones no es tan sencillo. Al sistematizar las experiencias, incluyendo las observaciones de campo, los registros de diarios y las entrevistas, bajo el marco de las experiencias relacionadas a las enfermedades y la muerte en el contexto hospitalario, los médicos han explicado ampliamente la importancia del "tipo" de enfermedad. Debido a que existen enfermedades que no tienen cura, y el desarrollo "natural" de ésta devendrá en la muerte.

Será este tipo de esenarios los que complejizará la relación entre el personal de salud, la familia y los pacientes, ya que este triángulo, donde la enfermedad no tiene una "cura" como tal, sino que la terapéutica implica la ejecución de una serie de cuidados paliativos encaminados a disminuir los malestares de la propia enfermedad, provoca que los familiares y pacientes no acepten con facilidad que este sea el único camino. Por el contrario, se anteponen los caracteres simbólicos e imaginarios de la institución hospitalaria como espacio del saber, donde la figura del médico encarna la máxima autoridad de esta institución, es decir, el médico siempre debe saber curar al paciente.

Es alrededor de estos componentes simbólicos e imaginarios, elementos que se configuran a partir de una serie de supuestos y apariencias socio-históricamente construidas, que se le otorga a la praxis médica, una serie de significaciones y demandas sociales como la curación y el salvar vidas. No obstante, esta demanda hacia el médico, el personal de salud y el hospital, aun enmascarada por la expectativa de "curación", apunta a la pregunta sobre cómo postergar la muerte, cómo evitarla, cómo adormecer el dolor, en otras palabras, en la construcción de los imaginarios sociales de lo que debe hacer un médico y el hospital ante la enfermedad, se niega a la muerte misma. Esta situación, producirá un malentendido y tensión entre las partes sin igual.

Esta gran tensión, sin duda alguna está articulada con las formas en que hemos construido como sociedad conceptos como enfermedad, cuerpo, vida, curación, dolor y muerte. Todos ellos atravesados y configurados por los principios de la modernidad, de la ciencia y de la clínica. En ese sentido, el hospital, se presenta como espacio imaginario y simbólico de entrecruzamiento de anhelos, fantasías, expectativas, historia, funciones y relaciones sociales, que se aglutinan y corporizan en él.

De este modo, el hospital se configura como un determinado dispositivo para atender ciertas urgencias sociales relacionadas a la salud y a la enfermedad. Todo ello, lleva a las personas comunes, a identificar al hospital como "la institución clínica" que busca a toda costa una terapeútica que redunde en la curación de la enfermedad y en el adormecimiento del 
dolor, negando la posibilidad de la muerte.

\section{Con un pie en la ciencia y otro en la religión}

El subtítulo de esta sección pertenece a una frase de un médico residente de tercer año de medicina interna, quien compartía sus impresiones sobre su trabajo en el área de Unidad de Cuidados Intensivos. Este médico de 28 años condensó su experiencia en la frase para manifestar el conflicto con el que vivía cada vez que debía tener la "charla" con algún familiar de un paciente agónico. Las situaciones más usuales se trataban de casos de personas que padecían alguna enfermedad crónica-degenerativa, ${ }^{10}$ y/o por su avanzada edad -mayores de 70 años-, ya no estaban en condiciones físicas de resistir más intervenciones encaminadas a salvar su vida. Por lo que debía informar a los familiares sobre la difícil situación de su paciente y así tomar una decisión sobre los siguientes pasos a seguir.

Sin embargo, cuando los médicos informaban esta situación desafortunada a los familiares, éstosnoaceptaban fácilmentelas propuestas de intervención. Lascuales generalmente son: 1) continuar con la intervención a sabiendas de que no habrá una mejoría sino que todos los esfuerzos estarán dirigidos en otorgar cuidados analgésicos para la disminución del dolor y disminución de la sintomatología; 2) el retiro paulatino de la intervención terapéutica sobre el paciente o la llamada "Limitación del Esfuerzo Terapéutico", que conlleva a que la enfermedad siga el único curso posible: la muerte; 3) o finalmente el "Alta de Máximo Beneficio", que implica llevar al paciente a su casa para que muera allí. ${ }^{11}$

Ante esta situación los familiares manifiestan su enojo y frustración hacía el personal de salud. En su opinión y perspectivas, los médicos no hacen "lo suficiente" para salvar a su paciente. Reiteradamente demandan "hacer todo lo posible", deben "salvarlo".

Al analizar esta situación, los profesionales de la psicología podemos hacer una lectura teórica de los que está sucediendo. Inmediatamente podemos explicarlo a partir de las etapas del duelo, ${ }^{12}$ propuestas por Elisabeth Kübler-Ross (1993). Los familiares están pasando por la primera fase de negación y pasando por el enojo hacía la negociación. Esto es, en primera instancia no asumen la resolución inevitable de la muerte, e inmediatamente después inician la negociación: un mejor tratamiento, que lo atienda otro médico, seguramente no están haciendo su máximo esfuerzo.

No obstante, esta explicación desde la psicología puede redundar en una postura reduccionista que termina por individualizar la complejidad de la trama social e histórica que se están entretejiendo en esta situación. Es importante señalar que todos estos procesos psíquicos -como el duelo-, están atravesados por dispositivos e instituciones sociohistóricas como la tecnología y la ciencia que tienen como propósito poner un velo sobre la muerte.

Por lo anterior, se podría entender que los procesos de negación no obedecen a

10 Por ejemplo, diabetes, hipertensión, patologías renales, cardiacas, cuadros respiratorios.

11 Valga el eufemismo...

12 Las ya conocidas: negación, ira o enojo, negociación, depresión y aceptación (Kübler-Ross, 1993), etapas descriptivas que tienen su correlato psicodinámico en la posición del duelo. 
experiencias personales o patologías de la persona que le impiden procesar la muerte de su ser querido, sino que se trata de una negación proveniente de una construcción social en donde la muerte misma no tiene lugar en la sociedad, y mucho menos en la institución hospitalaria, esto es, al hospital se va a recuperarse, no a morir, con lo que se invierte la ecuación pre-moderna.

De igual forma, el lugar donde se depositará el enojo y frustración de los familiares es sobre el personal de salud y el hospital. Se los concibe como incapaces de darle una solución encaminada hacia la vida y la curación, por lo que se interpreta como ineptitud o fracaso. En ese sentido, en el hospital que se condensan los imaginarios del "todo es posible", de la victoria de la vida sobre la muerte, lo cual produce un malentendido entre las expectativas del personal de salud y los familiares-pacientes. Los familiares y pacientes no pueden aceptar que el único escenario posible sea la muerte, se demanda que el personal de salud "haga algo más", algo que salve la vida del otro.

Se inicia una negociación entre las partes, donde -en el mejor de los casos- el personal de salud termina por acompañar proceso de asimilación, simbolización, duelo y aceptación de la familia. Cabe señalar, que aun cuando el personal de salud tiene cierta preparación para estas situaciones, la dinámica hospitalaria no siempre permite que este proceso se lleve de la mejor manera. Por un lado, debido a la carga de trabajo que realiza el personal de enfermería y médico, el cual no sólo consiste en la atención e intervención terapéutica del paciente, sino, además, del llenado de una serie de requisitos burocráticos que incluyen el llenado de formatos y del expediente del paciente, así como la expedición de notas y recetas, por mencionar algunos. Por el otro, porque no todo el personal de salud cuenta con una capacitación formal sobre los procesos de acompañamiento en el duelo. ${ }^{13}$

Bajo este contexto, se espera que el personal que brinde la atención especializada sobre el procesamiento del duelo, la simbolización de la experiencia y un acompañamiento psicoterapéutico sea el personal de psicología del hospital. No obstante, a través del registro de observaciones y entrevistas de los tres hospitales en donde se intervino e investigó (ISSSTEP, HUP y el Hospital General de Tepeaca), lo que se destaca es que no se cuenta con el personal mínimo para brindar atención psicológica en situaciones de procesos agónicos y muerte.

Considerando que el psicólogo hospitalario, se ocupa de problemáticas relacionadas al estrés y salud en pacientes ambulatorios y hospitalizados; la intervención psicológica en el tratamiento del dolor crónico; la intervención psicológica en los pacientes oncológicos; los programas de rehabilitación; preparación e intervención en pacientes quirúrgicos; y para la preparación y selección de candidatos para trasplante de órganos (Soriano Pastor et al., 2009). ${ }^{14}$

A partir de la sistematización de experiencias de los tres hospitales, se detectó que, el poco personal de psicología está destinado a brindar consultas externas a los pacientes que

13 De los casos observados y registrados, los médicos y enfermeras no tienen capacitación formal para tratar este proceso de acompañamiento. Sino que se reduce a una clase en licenciatura o algún curso corto sobre el tema. No es una generalización, pero si es una constante en las entrevistas realizadas. La mayoría del personal tuvo que buscar una capacitación extra, pero valoran que no son especialistas en este campo y que debería ser el personal de psicología quien realice esta actividad.

14 Por mencionar algunas de las funciones del psicólogo hospitalario, las funciones y roles de éste, pueden abarcar un abanico de tareas. Cfr. Soriano Pastor et al. (2009). 
han sido dados de alta, pero que el personal médico consideró que requería algún tipo de intervención psicológica. Así, los psicólogos sólo brindan atención a pacientes ambulatorios con diagnósticos relacionados a algún trastorno psíquico (depresión, ansiedad, estrés). Pero, en los casos de procesos agónicos, de adherencia a los tratamientos, de enfermedades crónico-degenerativas y terminal, como en el caso de muertes y duelo, no son los psicólogos, - personal especialista en estos procesos-, sino el personal de enfermería, médico y de trabajo social, quien termina resolviendo como puede.

En esta situación compleja, cuando los médicos comienzan a plantear las alternativas de limitación del esfuerzo terapéutico, el paciente y la familia no quieren ser parte de la toma de decisiones, sino que se espera - de nuevo sostenido por el imaginario de la institución hospitalaria- que sea el médico quien decida, o en última instancia, Dios. Constantemente le reiteraban a Mayleth que no era posible "cesar la atención". Atravesados por la "culpa no hacer lo suficiente", insistían que ellos no podían tomar semejante decisión, que se trataba de una decisión que debía tomar el médico como profesional de la salud, o, por otro lado, Dios tenía la decisión final.

Por ello, el joven residente dijo "estamos con un pie en la ciencia y con otro en la religión", en el contexto de la mediación que debían hacer para sostener la vida de los pacientes. Es decir que, mientras, hacen todo lo que es técnicamente posible, esperan que la enfermedad siga su desenlace final como parte de un plan místico o espiritual a cargo de Dios.

Cuando se enfrentaban a estas situaciones, de manera generalizada el personal de salud se sentía conflictuado por esta negociación. En su perspectiva, los familiares debían tomar la decisión de llevarse a su paciente a morir a casa, o al menos de retirar paulatinamente la intervención, ya que, en su opinión, se sometía al paciente a procesos agónicos extendidos, dolorosos y muy costosos (económica y emocionalmente), no sólo para el propio paciente, sino para los familiares y para el propio personal de salud.

Cada una de las partes tenía su postura clara frente a la decisión que debía o no tomarse. Lo que tenían en común, era que nadie quería hacerse cargo del moribundo.

\section{Reflexiones Finales}

Las enfermedades terminales, que implican procesos dolorosos, de agonía y muerte tienen rasgos singulares que consideramos se deben analizar más a fondo. En la instituciónhospital, los procesos agónicos y en los casos en que no se utiliza toda la tecnología o se hace el intento de reanimación, la muerte suele vivirse como una falta de la institución y del equipo de salud (Emmer y Schejter, 2014), y no como un desenlace esperado de una enfermedad incurable o de la propia vida. Se manifiesta el semblante de la negación de la muerte en nuestra sociedad actual.

Aunado a eso, de parte del personal de salud, siempre existe el riesgo latente de un juicio de mala praxis y que a veces es reforzado por amenazas concretas "sé que se va a morir, pero que no sea por descuido de ustedes". Esto condiciona la continuación de la intervención 
sobre el cuerpo del paciente, independientemente de la desproporción del recurso utilizado en relación a los beneficios posible a lograr. El despliegue de la tecnología y de los procedimientos terapéuticos complejos es un factor que retroalimenta las fantasías de curación y termina por obturar la elaboración de la pérdida en la familia (Emmer y Schejter, 2014, s. p.).

Es entonces que se genera la creencia de que todo depende de la tecnología, que por supuesto es mediada por su máximo representante: el médico. Otras alternativas como cuidar o acompañar suelen ser consideradas como equivalentes a no hacer nada y despiertan la sensación de que no se les ofreció "lo mejor" (Emmer y Schejter, 2014). Se pierde así una de las funciones fundamentales del equipo de salud que es el "cuidar" y que tiene una resonancia con su pasado de hospicio.

Lo más moderno, lo más costoso, tranquiliza los sentimientos de impotencia y culpa. Cuidar es, algunas veces, preservar al paciente de la "maquinaria médica" que por momentos parece autonomizarse del pensamiento y tornarse imparable. Queda así poco lugar para acercarse a la muerte por fuera de los caminos ofrecidos por un modelo médico tecnificado (Emmer y Schejter, 2014; Illich, 1975; Elías, 2015). En ese sentido, el pasado del hospital en su figura del hospicio se encuentra negado. Aquellos momentos en donde el alivio espiritual eran parte de la atención final de la vida se muestran olvidados, a pesar de que, en la actualidad, existen las figuras de los "pabellones tanáticos" o del hospicio en algunos países, los cuales buscan "llevar al máximo la calidad de la vida que le reste al paciente" (Sherr, 1992, p. 3). En la mayoría de los países no encontramos lugares destinados para llevar los procesos agónicos o terminales. ${ }^{15}$

Por otro lado, tampoco se pone en juego la propia elaboración del duelo de parte del personal de salud en el trabajo con pacientes graves, crónicos o terminales ante los que fracasan los métodos terapéuticos. Tal y como los destaca Darian Leader (2014), la elaboración del duelo siempre se realiza socialmente. Debido a que, en todo duelo, se pasa por un proceso de identificación que se configura a través de las formas culturales existentes para darle sentido a la muerte. Por ello, cualquier muerte, aunque no sea necesariamente de alguien conocido o muy cercano, termina por revivir aquellos duelos que sí lo son. $O$ al menos, termina por enfrentarnos con aquellas instituciones y dispositivos que se encargan de atribuirle sentido y de simbolizar la muerte. Por ejemplo, las instituciones eclesiásticas y religiosas, que históricamente se han encargado de significar estas experiencias.

De ahí que el pasado religioso del hospicio resulte interesantísimo, ya que, en el pasaje a la institucionalización del hospital, las formas de significación de la muerte y de la enfermedad dejan de asociarse con los esquemas religiosos, pero pasan a ser interpretados desde los marcos científicos que son insuficientes para simbolizar y procesar la cara inexplicable de la muerte. Al final, tal y como lo dijo el joven médico terminan con "un pie en la ciencia y otro en la religión".

Por si fuera poco, el personal de salud no sólo se confronta con sus propios duelos, a la

15 El proceso de secularización de la sociedad no ha generado una respuesta de significación frente a la muerte. Los sujetos modernos se encuentran desamparados frente a ella. La negación es tanto más fuerte cuanto la necesidad urgente de encontrar un sentido, que las significaciones modernas no proveen. Por ello, ese "pie en la religión" no es únicamente un asidero imaginario del médico, sino un soporte fundamental del dispositivo asistencial, que tiene que recurrir a estas significaciones premodernas para sostenerse. 
muerte o la "no-curación" de un paciente, sino también al duelo por su esfuerzo y su esperanza frustrados. La desilusión suele traer aparejada la sensación de agotamiento y de desinterés. "Se extingue el fuego, burn out, de la pasión que da sentido al esfuerzo y al trabajo" (Emmer y Schejter, 2014, s. p.). En ese sentido, el establecimiento se queda corto en los elementos necesarios para la simbolización de la muerte, bajo los esquemas de la modernidad y la técnica, la muerte no tiene lugar en el hospital. ¿No es esta una contradicción no sólo de la medicina, sino de nuestra sociedad? Dice Norberto Elías:

Jamás anteriormente ha muerto la gente de una manera tan poco ruidosa y tan higiénica como hoy en día en este tipo de sociedades, y jamás lo ha hecho en unas condiciones que hayan fomentado tanto la soledad (Elías, 2015, p. 105).

¿Qué no podríamos sospechar que se trata del proyecto moderno? Recordemos que las técnicas de la medicina son las que constituyen al sujeto moderno, la primera: la mirada. La cual "tiene la paradójica propiedad de entender un lenguaje en el momento en que se percibe un espectáculo, [...] en la clínica, lo que se manifiesta es originariamente lo que se habla." (Foucault, 2014, p. 150).

Por consiguiente, es de vital importancia el momento en que se cambia la forma de percibir a la muerte en medicina, donde el mismo Bichat habla de que más allá de liberar a la medicina del miedo de la muerte, se ha integrado esta muerte a un conjunto técnico y conceptual. El efecto es que la muerte se conciba como parte de un proceso, pero de un proceso lógico, un espacio que sí es accesible para el ser humano. Al mismo tiempo que se concibe entonces como un espacio accesible, se le niega, queda fuera de las instituciones modernas.

El hacer "accesible la muerte" para la ciencia, no se trata más que de un espejismo, un semblante, un imaginario de un espacio accesible para el ser humano. Es así que el discurso de las ciencias es una consecuencia lógica y directa del proyecto de la modernidad y que simultáneamente lo legitima, respalda y reivindica; y en particular lo hace a través del discurso de la clínica, en éste se materializa, ya que se interviene sobre el cuerpo. Es la ilusión de controlar, asir y predecir a la muerte. El imaginario del control sobre la institución de la muerte.

Con lo anterior, imaginemos la escena: la familia, el paciente agónico y el personal de salud. Desde la perspectiva de la familia y el paciente no cuentan con la información adecuada ni suficiente sobre lo que tiene su paciente, lo cual produce una serie de tensiones con el personal de salud. La familia no sabe qué hacer con su moribundo, no quiere llevárselo a casa. ¿Y cómo hacerlo si la muerte ha quedado negada de todo lugar social? Están en su derecho de no aceptar el "Alta de Máximo Beneficio", pero simultáneamente y del otro lado de la relación, los médicos tampoco se quieren hacer cargo del paciente moribundo. ¿Es entonces responsabilidad del médico hacerse cargo de la muerte cuando ésta se encuentra forcluida de la institución? Es una situación en donde el profesional de salud posee el conocimiento, el know-how de la técnica sanitaria. 
La sociedad llamada del bienestar y la abundancia ha descubierto que no puede mostrar abiertamente su rostro de violencia sin ocasionar en el seno de sí misma el nacimiento de unas contradicciones demasiadas evidentes, que terminarían por volverse contra ella. Por ello, ha encontrado un nuevo sistema: extender la concesión del poder a los técnicos que lo ejercerán en su nombre, y seguirán creando -a través de otras formas de violencia-: la violencia técnica [...] El nuevo psiquiatra social, el psicoterapeuta, el asistente social, el psicólogo de empresas, el sociólogo industrial, son únicamente los nuevos administradores de la violencia del poder, en la medida en que -suavizando asperezas, disolviendo resistencias, resolviendo conflictos engendrados por las instituciones-, se limitan a permitir, mediante su acción técnica aparentemente reparadora y no violenta, la perpetuación de la violencia global (Basaglia, 1972, p. 132).

¿Pero qué acaso la tecnificación no los conflictúa y los afecta? ¿El personal de salud queda exento de ser objetos de la violencia que se ejerce? Lourau hace mención de la distancia institucional, para explicar el fenómeno en el cual las "técnicas" dan un significado que abarca el conjunto de las operaciones e instrumentos utilizados como medio en la práctica social. "Se hablará de distancia práctica para designar esa forma de no saber referente a la función del sustrato material de todas las instituciones y de la organización social" (Lourau, 1991, p. 268).

Por otro lado, Franco Basaglia (1972) menciona que el enfermo al recibir "la terapéutica" se convierte en un cuerpo institucionalizado, se vive como objeto de violencia. Empero, ¿el médico no es también un cuerpo institucionalizado? ¿No es también objeto de la violencia global que se ejerce a través de la técnica? Y aún peor, quedan inmersos en la lógica instituida de la violencia global y de la técnica. ¿No se tendría que poner en cuestión la propia noción de vida que se construyó en la medicina? ¿Y con la vida, la muerte, la salud y la enfermedad? ¿Por qué es tan diferente la manera de afrontar la muerte de la persona desde el lugar del familiar al del médico? ¿No es el duelo una elaboración colectiva? Si no, ¿por qué es tan conflictuante para ellos la agonía de otro cuerpo frente al suyo?

Cabría preguntarse ¿quién decide quien vive y quién muere? ¿Realmente es la familia o el paciente? ¿O se trata del médico? Regresemos a las situaciones ya narradas anteriormente: los médicos y los familiares quedan en lugares no muy claros con respecto a quién es el que toma la decisión de seguir dando atención terapéutica para prolongar la vida. Empero, el médico no es un juez que decide, sino que basa la decisión en una serie de presupuestos, protocolos, el juramento Hipocrático claro está. En ese sentido no es el médico como tal quien toma la decisión, sino que esta está basada en el carácter universal de la institución médica, la cual niega los procesos de muerte como parte de los procesos de vida.

Es así que lo que emerge son los procesos de institucionalización del hospital, los cuales, han devenido en un entendimiento del hospital como un terreno imaginario y simbólico que en su búsqueda de la curación de la enfermedad, en el adormecimiento del dolor y su combate contra la muerte, a través de las técnicas y tecnologías modernas, que terminan por establecer un régimen de cuidado tecnocrático que trata de invisibilizar la muerte (López Varela, 2019; Emmer y Schejter, 2014). 
Sin duda alguna, la invitación del presente texto busca cuestionar, problematizar y abrir vetas sobre las formas en que estamos relacionándonos como sociedad con la muerte. Con la intención, de preguntarnos ¿cómo convivimos con ella? ¿Es posible cambiar nuestra relación con la muerte? ${ }^{16}$

\section{Referencias Bibliográficas}

BASAGLIA, Franco. La institución negada. Buenos Aires: Barral Editores, 1972. 248 p.

BERGER, John. Con la esperanza entre los dientes. Buenos Aires: Alfaguara, 2011. 168 p.

BERNARD, Marcos. Las condiciones del grupo acción. En: LAPASSADE, G. et al. (comp.). Análisis institucional y socioanálisis. Distrito Federal, México: Nueva Imagen, 1977. p. 241-264.

CASTORIADIS, Cornelius. La institución imaginaria de la sociedad. Barcelona: Tusquets, 1983, $265 \mathrm{p}$.

CURSO DE SISTEMATIZACIÓN DE EXPERIENCIAS. Manual de las y los participantes. 3ㅇ. ed. Ciudad de México: Sedepac, 2015. 142 p. Disponible en: <http://indesol.gob.mx/cedoc/ pdf/I.\%20SOCIEDAD\%20CIVIL/Sistematizaci\%C3\%B3n\%20de\%20Experiencias/Curso\%20 de\%20sistematizaci\%C3\%B3n\%20de\%20expereincias.\%20Manual\%20de\%20las\%20y\%20 los\%20participantes.pdf>. Acceso en: 16 dic. 2019.

DONZELOT, Jacques. La policia de las familias. Buenos Aires: Nueva Visión, 2008. 223 p.

ELÍAS, Norbert. La soledad de los moribundos. México: Fondo de Cultura, 2015. 139 p.

EMMER, Silvia y SCHEJTER, Virginia. Ética y Lazo Social. En: MORA LARCH, Francisco. Proceso Grupal. [S.l.], 4 nov. 2014. Disponible en: <http://procesogrupal.overblog.com/2014/11/eticay-lazo-social-de-silvia-emmer-y-virginia-schejter.html>. Acceso en: 20 nov. 2019.

FOUCAULT, Michel. La vida de los hombres infames. Traducido por Julia Varela y Fernando Álvarez-Uría. La Plata: Altamira, 1996. 223 p.

FOUCAULT, Michel. Historia de la locura en la época clásica I. México: Fondo de Cultura Económica, 1998. 125 p.

FOUCAULT, Michel. El poder psiquiátrico. Buenos Aires: Fondo de Cultura Económica, 2007. $448 \mathrm{p}$.

FOUCAULT, Michel. Historia de la sexualidad 3. La inquetud de sí. Ciudad de México: Siglo XXI, 2013. 269 p.

FOUCAULT, Michel. El nacimiento de la clínica. Buenos Aires: Fondo de Cultura Económica, 2014. 272 p.

16 Estas reflexiones, indudablemente, no sólo ponen en cuestión la perspectiva de la vida que se desprende de la institución médica y clínica, sino también sobre la enfermedad. Convierte a ésta en una figura antagónica de la salud, y su definición del cuerpo anula los procesos colectivos. Las consecuencias son clarísimas en la manera de enfrentar las epidemias... 
FRESQUET FEBRER, José Luis. Adolph Gubler y el Journal de Thérapeutique. Asclepio. Revista de historia de la medicina y de la ciencia, 45, 2, p. 143-186, 1993.

GIRARD, René. La violencia y lo sagrado. Barcelona: Anagrama, 2005. 337 p.

GUBER, Rosana. El salvaje metropolitano: Reconstrucción del conocimiento social en el trabajo de campo. México: Paidós, 2005. 227 p.

ILLICH, Iván. Némesis Médica: La expropiación de la salud. México: Breve Biblioteca de Respuesta-Barral, 1975. 218 p.

KÜBLER-ROSS, Elisabeth. Sobre la muerte y los moribundos. Barcelona: Grijalbo, 1993. 360 p.

LE BOUHELLEC, Laurence. ¿Cómo relacionarse con el arte evitando las trampas de la subjetividad estética maquínica? Entorno UDLAP. Puebla, n. 3, p. 36-49, sep. 2017. Disponible en: <https:// entorno.udlap. $\mathrm{mx} /$ revista-3-articulo-4-como-relacionarse-con-el-arte-evitando-lastrampas-de-la-subjetividad-estetica-maquinica/>. Acceso en: 16 dis. 2019.

LEADER, Darian. La moda negra: Duelo, Melancolía y depresión. Madrid: Sexto Piso, 2014. 195p.

LÓPEZ VARELA, Guillermo. Hacer de la muerte una pregunta: Subjetividades antagónicas en el capitalismo como religión y entramados culturales de vida en contextos de la Santa Muerte en Puebla (México). 2019. Tesis (Doctor en Sociología) - Instituto de Ciencias Sociales y Humanidades "Alfonso Vélez Pliego", Benemértia Universidad Autónoma de Puebla, Puebla, 2019. $374 \mathrm{p}$.

LOURAU, René. El análisis Institucional. Buenos Aires: Amorrurtu, 1991. 297 p.

MATAMOROS PONCE, Fernando. Tradición y rebelión en el imaginario: Un acercamiento a las estructuras simbólicas en el tiempo de la historia y lo religioso. Bajo el Volcán. Puebla, v. 4, n. 8, p. 185-209, dic. 2004. Disponible en: <https://www.redalyc.org/pdf/286/28640812.pdf>. Acceso en: 05 oct. 2019.

RIFKIN, Jeremy. El sueño europeo. Barcelona: Paidos, 2004. 528 p.

SHERR, Lorraine. Agonía, muerte y duelo. México: Manual Moderno, 1992. 273 p.

SORIANO PASTOR, José et al. Guía práctica de Psicología de la Salud en el ámbito hospitalario. Valencia: Collegi Oficial de Psicologia de la Comunitat Valenciana, 2009. 174 p.

Recebido em: 18 de dezembro de 2019

Aprovado em: 12 de abril de 2020 